\title{
ARTí́culo
}

\section{Efectos del lavado in situ de redes en sedimentos asociados a la acuicultura en el sur de Chile}

\author{
Effects of in situ washing nets in sediment associated with \\ aquaculture in southern of Chile
}

\begin{abstract}
Rodrigo Vera ${ }^{1}$ y Ana María Vergara ${ }^{2}$
'Departamento de Medio Ambiente, División de Acuicultura, Instituto de Fomento Pesquero, Balmaceda 252, Puerto Montt, Chile. rodrigo.vera@ifop.cl

${ }^{2}$ Consultora independiente, Lunecke 1034, Parque Krahmer, Valdivia

Abstract.- The Chilean Environmental Regulation for Aquaculture provides the possibility of washing nets as bio-fouling control in situ and considering the growth of the salmon farming industry in recent years, it is imperative to generate the necessary and relevant information to diagnose different cleaning systems in situ and their potential environmental impact. Accordingly, sampling campaigns were conducted in 3 sectors of the Los Lagos Region, where sediment rates and organic material was determinated in water and sediment, pre and post washing nets. The results show differences in the matrices studied between 3 sites, but only differences within Pichagua (younger before after washing in situ) y Alao Sur (mayor before after washing) in sedimentation rates.
\end{abstract}

Key words: Nets, washing in situ, sediment, aquaculture

Resumen.- El Reglamento Ambiental para la Acuicultura chilena establece la posibilidad de realizar lavado de redes in situ como medida de control de bio-incrustantes y considerando el crecimiento del sector salmonero durante los últimos años, es imperativo generar la información necesaria y relevante que permita diagnosticar los distintos sistemas de lavado in situ y su potencial impacto ambiental. Se realizaron campañas de muestreo en 3 sectores en la región de Los Lagos, donde se obtuvieron muestras de agua y sedimento mediante un diseño de muestreo que permitió comparar valores de materia orgánica particulada y sedimentable previo y posterior a la actividad de lavado de redes in situ sin retención. Los resultados muestran diferencias en las matrices estudiadas entre los 3 sitios, pero solo diferencias dentro de Pichagua (menor antes que después del lavado in situ) y Alao Sur (mayor antes que después del lavado) en las tasas de sedimentación.

Palabras clave: Redes, lavado in situ, sedimentos, acuicultura

\section{INTRODUCCIÓN}

Una de las principales dificultades que enfrenta la industria del salmón en el ámbito de la producción corresponde a la adherencia de material biológico denominado incrustantes o adherencia viva. Los incrustantes se pueden definir como una sucesión ecológica que resulta en la formación de comunidades de algas e invertebrados marinos instalados sobre diferentes tipos de sustratos relacionados con actividades marítimas (Green 2007).

El crecimiento de material incrustante genera 3 principales efectos negativos directos sobre las jaulas de peces. Primero, es capaz de provocar una deformación de la red y generar fatiga de material debido al peso extra que está conteniendo la estructura (Fitridge et al. 2012). También puede restringir el intercambio de agua debido a la obstrucción del flujo a través de la red (30 a 40\%) (Beveridge 1987), que en sistemas de cultivos intensivos tiene como consecuencia la pérdida de calidad del agua, ya que disminuye los niveles de oxígeno disuelto y de la tasa de eliminación del exceso de alimento y de otros residuos. Finalmente, se considera que incrementa la vulnerabilidad a enfermedades debido a que las comunidades de organismos incrustantes actúan como un reservorio de patógenos, que sumado a los pobres niveles de oxígeno y al estrés que esto genera en los peces, estarían actuando como facilitador de enfermedades (Fitridge et al. 2012) (e.g., Caligus, SRS, ISA).

Para el control de los bio-incrustantes se emplean desde la década de 1980 diversos enfoques: (1) cambio y limpieza de redes para remover los organismos incrustantes y mantener el intercambio de agua, (2) anti-incrustantes químicos tales como compuestos de cobre para evitar el reclutamiento de organismos incrustantes, y más recientemente (3) control biológico utilizando peces o invertebrados herbívoros que ramoneen bioincrustantes de la superficie de las redes.

Focalizándose sólo en el cambio y limpieza de redes para el control de incrustantes, se reconoce que los acuicultores de regiones templadas y tropicales cambian o limpian jaulas con 
frecuencia para mantener el intercambio de agua cuando las cargas de incrustantes así lo determinan. Esto se realiza entre 5 a 8 días en verano en Australia (Hodson \& Burke 1994), de 8 a 14 días en Japón, 14 días en Malasia (Lee et al. 1985) y de 3 a 4 semanas en Canadá (Menton \& Allen 1991). Si la distribución de las incrustaciones se limita a la zona superior de la jaula, la frecuencia de la limpieza puede ser ampliada y en su reemplazo se elevan las redes unos pocos metros fuera del agua para permitir el secado de los incrustantes y su posterior desprendimiento (Needham 1988).

El cambio de redes involucra un importante costo para la industria, requiriendo la compra de un gran número de redes y la prestación de servicios de limpieza. Más aún, el cambio frecuente de redes involucra riesgos al producirse daños o pérdida de los stocks de peces, así como perturbaciones en los regímenes de alimentación que pueden disminuir las tasas de crecimiento. El cambio de redes es un trabajo intensivo que involucra traslado a tierra, secado, seguido de limpieza con agua a alta presión o lavadoras automáticas (Cronin et al. 1999). Tanto el procedimiento de lavado como la manipulación frecuente dañan las redes y reduce la vida media de éstas.

Como una alternativa al recambio de redes ha surgido la limpieza in situ sin retención, principalmente con hidrolavadoras con discos que impulsan agua a presión. Estos sistemas son de uso generalizadoen varios países como Australia (Hodson et al. 1997) y Noruega (Guenther et al. 2009). Según Olafsen (2006), más de la mitad de las salmoneras en Noruega realizan regularmente limpieza in situ sin retención. Aunque esta limpieza mecánica frecuente es cara en estos países, la combinación de ésta con otras estrategias puede reducir los costos de control de incrustación biológica hasta un $50 \%$ por $\mathrm{m}^{2}$ de red (www.crabproject.com).

La limpieza in situ está casi totalmente automatizada y es la estrategia dominante en las grandes empresas internacionales (Fitridge et al. 2012). Sin embargo, persiste el problema de que la materia orgánica (MO) desprendida desde las redes, cae invariablemente al cuerpo de agua y una fracción, que no puede ser removida, permanece en las redes (Greene \& Grizzle 2007) la cual puede volver a crecer rápidamente (Guenther $e t$ al. 2010). El proceso de lavado también puede desencadenar la liberación de larvas que conduce a una rápida recolonización de las redes (Carl et al. 2011), así como a la fragmentación y nuevo crecimiento de algunos organismos coloniales (Carl et al. 2011, Hopkins et al. 2011). La limpieza in situ por lo tanto, debe ser un proceso frecuente, es decir, los restos de materia orgánica desprendida de las redes se dispersan en el agua debido a su pequeño volumen, sólo si la limpieza in situ se realiza principalmente en las primeras etapas de la sucesión biológica (Fitridge et al. 2012).
Si se consideran factores como el crecimiento del sector salmonero en Chile durante los últimos años y las dimensiones de las balsas jaulas y redes loberas empleadas en la actualidad (aumento del número de peces en el agua), las actividades de lavado in situ sin retención de sólidos y por último el aporte de materia orgánica ya existente producto de la actividad natural y antrópica del centro de cultivo (por el alimento no consumido y fecas), es imperativo generar la información necesaria y relevante que permita caracterizar los distintos sistemas de limpieza in situ utilizados en Chile y su impacto en los sedimentos asociados a la acuicultura. De acuerdo a esto, se plantea la hipótesis de que los organismos incrustantes desprendidos desde las redes por el lavado in situ, se depositan bajo los centros de cultivo y debido a esto, existen diferencias en las concentraciones de materia orgánica total antes y después del lavado in situ de redes en sedimentos y columna de agua de las zonas de Pichagua, Huelmo y Alao Sur, en el Archipiélago de Chiloé, sur de Chile.

\section{MATERIALES Y MÉTODOS}

Para determinar tasas de sedimentación y materia orgánica en la columna de agua y en sedimentos antes y después de realizar lavado in situ de redes asociado a la salmonicultura y evaluar el impacto que este tipo de estrategias tiene en los sedimentos bajo los centros de cultivo, se muestrearon 3 localidades en el sur de Chile. Centro 1 Huelmo (41 $41^{\prime} 7,37^{\prime \prime} S$;

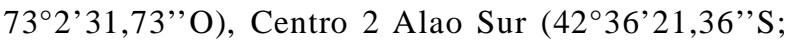
$\left.73^{\circ} 19^{\prime} 15,29^{\prime \prime} \mathrm{O}\right)$ y Centro 3 Pichagua (4310'41,32's; $73^{\circ} 36^{\prime} 18,82^{\prime}$ 'O) (Fig. 1). Para la selección de estos lugares se utilizó el registro del Servicio Nacional de Pesca de Chile (SERNAPESCA), desde donde se calculó la fecha de instalación de la red, y considerando la periodicidad estacional del Reglamento Ambiental para la Acuicultura (RAMA), que centros deberían realizar lavado in situ de sus redes entre agosto y octubre 2012.

Las campañas en terreno se realizaron desde el 5 al 8 de octubre 2012 en Huelmo, del 28 al 31 de octubre 2012 en Alao Sur y del 23 al 26 de octubre 2012 en Pichagua.

Para captar el material orgánico particulado, en cada centro, se instalaron trampas de sedimento, en triplicado, en 6 estaciones de muestreo más una estación de referencia o control. La ubicación y distribución de las trampas dependió de las condiciones de corrientes de cada Centro, es decir, se instalaron en la dirección en que la corriente predominante arrastraría los incrustantes desprendidos durante el lavado, basado en información de la caracterización preliminar del sitio(CPS) de cada centro y de la marea imperante al momento del lavado. 

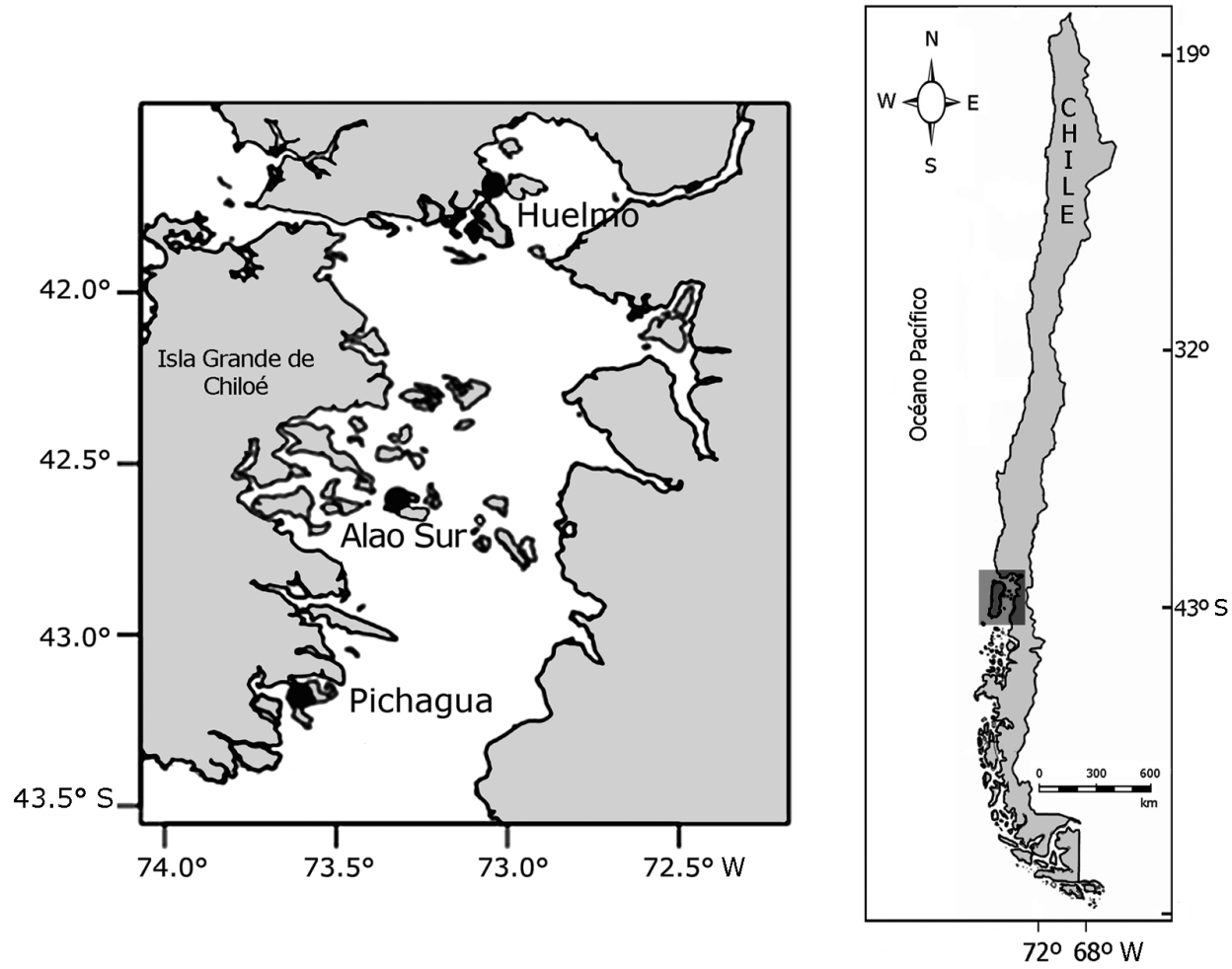

Figura 1. Ubicación de los centros de cultivo donde se realizó el experimento de cuantificación de material orgánico particulado / Location of the farms where the experiment for quantifying particulate organic material was carried out

La instalación de las trampas se realizó con el uso de un winche y la estabilidad de la trampa fue inspeccionada por un buzo autónomo de cada centro. La profundidad de la ubicación de la trampa fue de 10 a $12 \mathrm{~m}$, profundidad coincidente con la de lavado de las redes. El cambio de frasco entre el muestreo pre-lavado y post lavado también fue realizado por un buzo autónomo.

Las muestras obtenidas desde las trampas fueron trasladadas en cada frasco recolector de $1 \mathrm{~L}$, en forma vertical, en oscuridad y refrigerados $\left(2 \mathrm{a} 4^{\circ} \mathrm{C}\right)$ al laboratorio y mantenidos luego a $-4^{\circ} \mathrm{C}$ hasta el análisis.

Para evaluar la materia orgánica que ya había sedimentado se utilizó una draga de $0,1 \mathrm{~m}^{2}$ de mascada modelo Van Veen (Wildco) para obtener muestras del fondo marino, en las mismas 7 estaciones de muestreo de cada zona de estudio. De cada porción de sedimento obtenido con la draga, se tomaron 3 submuestras (pseudo réplicas) que fueron almacenadas en botellas plásticas de $250 \mathrm{ml}$ para su posterior análisis.
Se caracterizó cada una de las 7 estaciones de muestreo a través de la medición de variables oceanográficas. Se midió salinidad, temperatura y concentración de oxígeno disuelto, con el uso de un CTD-O (SAIV-AS) y se tomaron muestras de agua con botella Niskin a 2 profundidades ( 5 y $25 \mathrm{~m}$ ) para la estimación de seston orgánico. Estas muestras fueron almacenadas en frascos plásticos de $1 \mathrm{~L}$. Se estimó la profundidad de cada estación de muestreo con el uso de un ecosonda (GPSMap 420s Garmin).

Para establecer diferencias significativas entre las concentraciones de materia orgánica tanto en los sedimentos (trampas de sedimentos y dragas) como en la columna de agua, se usó test de Wilcoxon para muestras pareadas, (datos no poseen distribución normal, test de Shapiro-Wilk, $P_{\text {seston }}=9,769$ x $10^{-8}, P_{\text {dragas }}=5,968 \times 10^{-12}, P_{\text {trampas }}=3,419 \times 10^{-7}, P_{\text {granulometria }}=$ $\left.2,538 \times 10^{-14}\right)$. Los datos en porcentaje fueron transformados con arcoseno al realizar los test estadísticos (Zar 2010). 


\section{Resultados}

\section{Tasas de Sedimentación}

Las tasas de sedimentación, obtenidas desde las trampas de sedimentos instaladas en los centros estudiados, antes y después del lavado in situ se muestran en la Tabla 1. En Pichagua se determinaron tasas de sedimentación antes de la limpieza in situ con mediana de $0,89 \mathrm{~g} \mathrm{~m}^{-2} \mathrm{~d}^{-1}\left(0,36\right.$ a $\left.1,84 \mathrm{~g} \mathrm{~m}^{-2} \mathrm{~d}^{-1}\right)$, Huelmo $1,05 \mathrm{~g} \mathrm{~m}^{-2} \mathrm{~d}^{-1}\left(0,63\right.$ a $\left.5,52 \mathrm{~g} \mathrm{~m}^{-2} \mathrm{~d}^{-1}\right)$ y Alao Sur de $1,42 \mathrm{~g} \mathrm{~m}^{-2} \mathrm{~d}^{-1}\left(0,63 \mathrm{a} 2,05 \mathrm{~g} \mathrm{~m}^{-2} \mathrm{~d}^{-1}\right)$. Posterior a la limpieza in situ, la mediana de los valores observados de sedimentación fue de $1,15 \mathrm{~g} \mathrm{~m}^{-2} \mathrm{~d}^{-1}\left(0,63\right.$ a 1,94 $\left.\mathrm{g} \mathrm{m}^{-2} \mathrm{~d}^{-1}\right)$ para Pichagua, 0,89 $\mathrm{g} \mathrm{m}^{-2} \mathrm{~d}^{-1}\left(0,26\right.$ a $\left.2,10 \mathrm{~g} \mathrm{~m}^{-2} \mathrm{~d}^{-1}\right)$ para Huelmo y $1,10 \mathrm{~g} \mathrm{~m}^{-2} \mathrm{~d}^{-1}$ $\left(0,23\right.$ a $\left.1,78 \mathrm{~g} \mathrm{~m}^{-2} \mathrm{~d}^{-1}\right)$ para Alao Sur.
Al comparar las tasas de sedimentación de materia orgánica entre las áreas (test de Wilcoxon), se encontraron diferencias significativas entre la localidad de Pichagua y Alao $\operatorname{Sur}(P=$ 0,004). De a acuerdo a esto se analizaron de forma independiente las áreas en estudio, encontrándose diferencias significativas entre las tasas de sedimentación antes y después del lavado in situ para el área de Pichagua $(P=0,0243)$ con mayor sedimentación después del lavado in situ y Alao Sur $(P=0,048)$ con mayor sedimentación antes del lavado in situ, mientras que Huelmo no mostró diferencias significativas $(P=$ $0,088)$.

Tabla 1. Tasas de sedimentación, obtenidas desde las trampas de sedimentos instaladas en los centros estudiados, antes y después del lavado in situ / Sedimentation rates, obtained from sediment traps installed before and after in situ washing

\begin{tabular}{|c|c|c|c|c|c|c|c|}
\hline \multirow{2}{*}{ Estación } & \multirow{2}{*}{ Réplica } & \multicolumn{2}{|c|}{ Pichagua } & \multicolumn{2}{|c|}{ Huelmo } & \multicolumn{2}{|c|}{ Alao Sur } \\
\hline & & antes & después & antes & después & antes & después \\
\hline \multirow{3}{*}{1} & 1 & 1,841 & 1,210 & 1,947 & 0,579 & 1,210 & 1,105 \\
\hline & 2 & 0,894 & 1,263 & 1,263 & 0,737 & 0,631 & 1,157 \\
\hline & 3 & 0,368 & 1,263 & na & na & 1,157 & 1,105 \\
\hline \multirow{3}{*}{2} & 1 & 1,263 & 1,947 & 3,315 & 0,894 & 1,210 & 1,421 \\
\hline & 2 & 1,052 & 1,315 & 2,683 & 1,000 & 1,210 & 1,315 \\
\hline & 3 & 1,105 & 1,157 & 5,524 & 2,105 & 0,842 & 1,157 \\
\hline \multirow{3}{*}{3} & 1 & 1,000 & 1,315 & 2,894 & 1,526 & 1,736 & 0,263 \\
\hline & 2 & 0,947 & 1,789 & 1,368 & 1,421 & 1,315 & 1,789 \\
\hline & 3 & 0,842 & 1,000 & 1,315 & 1,578 & 1,263 & 1,000 \\
\hline \multirow{3}{*}{4} & 1 & 1,157 & 1,315 & na & na & 2,052 & 1,315 \\
\hline & 2 & 0,737 & 1,157 & na & na & 1,631 & 1,157 \\
\hline & 3 & 0,579 & 1,000 & na & na & 1,789 & 0,000 \\
\hline \multirow{3}{*}{5} & 1 & 0,894 & 0,737 & 1,052 & 0,737 & 1,789 & 0,737 \\
\hline & 2 & 1,000 & 1,105 & 0,631 & 0,894 & 1,368 & 1,105 \\
\hline & 3 & 0,684 & 1,000 & 0,842 & 1,894 & 1,526 & 1,000 \\
\hline \multirow{3}{*}{6} & 1 & 0,684 & 1,263 & 0,631 & 0,789 & 1,841 & 0,000 \\
\hline & 2 & 0,737 & 0,631 & 1,000 & 0,894 & 1,473 & 0,631 \\
\hline & 3 & 1,421 & 1,157 & 0,631 & 1,157 & na & 1,157 \\
\hline \multirow{3}{*}{7} & 1 & 0,894 & 1,105 & 0,789 & 0,737 & 1,526 & 1,105 \\
\hline & 2 & 1,105 & 0,631 & 1,000 & 0,263 & 1,684 & 0,631 \\
\hline & 3 & 0,842 & 1,789 & 0,737 & 0,474 & 1,421 & 1,684 \\
\hline \multirow{3}{*}{8} & 1 & 1,421 & 1,210 & & & & \\
\hline & 2 & 0,474 & 0,631 & & & & \\
\hline & 3 & na & na & & & & \\
\hline
\end{tabular}

na= no hay datos 
Tabla 2. Concentraciones de materia orgánica en la columna de agua antes y después del lavado in situ / Concentrations of organic matter in the water column before and after in situ washing

\begin{tabular}{|c|c|c|c|c|c|c|}
\hline \multirow{4}{*}{ Estación } & \multicolumn{6}{|c|}{ Concentración de seston en la columna de agua $\left(\mathrm{g} \mathrm{L}^{-1}\right)$} \\
\hline & \multicolumn{2}{|c|}{ Pichagua } & \multicolumn{2}{|c|}{ Huelmo } & \multicolumn{2}{|c|}{ Alao Sur } \\
\hline & $5 \mathrm{~m}$ & $15 \mathrm{~m}$ & $5 \mathrm{~m}$ & $15 \mathrm{~m}$ & $5 \mathrm{~m}$ & $15 \mathrm{~m}$ \\
\hline & antes después & antes después & antes después & antes después & antes después & antes después \\
\hline 1 & $0,00560,0028$ & $0,005 \quad 0,0064$ & $0,0060,0053$ & $0,005 \quad 0,0034$ & $0,017 \quad 0,0059$ & $0,0170,0073$ \\
\hline 2 & $0,00400,0036$ & $0,0050,0028$ & $0,0050,0033$ & $0,0040,0033$ & $0,0070,0045$ & $0,0040,0046$ \\
\hline 3 & $0,00400,0027$ & $0,0040,0021$ & $0,0030,0057$ & $0,0020,0058$ & $0,0060,0055$ & $0,01 \quad 0,005$ \\
\hline 4 & $0,00280,0029$ & $0,0030,0029$ & $0,0030,0041$ & $0,0050,0051$ & $0,0040,0045$ & $0,0050,0067$ \\
\hline 5 & $0,00270,0022$ & $0,0040,0021$ & $0,0050,0052$ & $0,0050,0036$ & $0,0050,0046$ & $0,0080,0035$ \\
\hline 6 & $0,00380,004$ & $0,003 \quad 0,002$ & $0,0040,0028$ & $0,0040,0025$ & $0,0050,0101$ & $0,0040,0078$ \\
\hline 7 & $0,00540,0038$ & $0,0020,0047$ & $0,0060,0033$ & $0,0040,0030$ & $0,0050,0046$ & $0,0080,0043$ \\
\hline 8 & $0,00470,0025$ & $0,003 \quad 0,0039$ & & & & \\
\hline
\end{tabular}

Tabla 3. Porcentaje de materia orgánica de sedimentos asociados a los centros de cultivo estudiados antes y después del lavado in situ / Percentage of sediment organic matter associated with farms studied before and after in situ washing

\begin{tabular}{|c|c|c|c|c|c|c|c|c|c|c|c|c|}
\hline \multirow{4}{*}{ Estación } & \multicolumn{12}{|c|}{ Porcentaje de Materia Orgánica en sedimentos (Draga) } \\
\hline & \multicolumn{4}{|c|}{ Pichagua } & \multicolumn{4}{|c|}{ Huelmo } & \multicolumn{4}{|c|}{ Alao Sur } \\
\hline & \multicolumn{2}{|c|}{ antes } & \multicolumn{2}{|c|}{ después } & \multicolumn{2}{|c|}{ antes } & \multicolumn{2}{|c|}{ después } & \multicolumn{2}{|c|}{ antes } & \multicolumn{2}{|c|}{ después } \\
\hline & Promedio & D. S. & Promedio & D. S. & Promedio & D. S. & Promedio & D. S. & Promedio & D. S. & Promedio & D. S. \\
\hline 1 & 1,168 & 0,077 & 0,971 & 0,136 & 1,102 & 0,075 & 1,190 & 0,048 & 1,686 & 0,131 & 1,363 & 0,079 \\
\hline 2 & 0,874 & 0,026 & 0,889 & 0,076 & 2,583 & 0,741 & 2,438 & 0,231 & 1,264 & 0,142 & 1,203 & 0,016 \\
\hline 3 & 0,954 & 0,048 & 1,101 & 0,137 & 2,069 & 0,350 & 2,890 & 0,184 & 1,311 & 0,026 & 1,534 & 0,172 \\
\hline 4 & 1,021 & 0,072 & 0,927 & 0,022 & 1,488 & 0,109 & 1,315 & 0,450 & 1,272 & 0,111 & 2,405 & 2,238 \\
\hline 5 & 0,922 & 0,183 & 1,271 & 0,535 & 1,225 & 0,367 & 1,795 & 0,435 & 1,177 & 0,417 & 1,876 & 1,364 \\
\hline 6 & 0,977 & 0,065 & 1,031 & 0,153 & 1,786 & 0,399 & 1,192 & 0,220 & 1,078 & 0,074 & 1,579 & 0,829 \\
\hline 7 & 1,107 & 0,048 & 1,024 & 0,216 & 1,451 & 0,417 & 1,220 & 0,070 & 1,107 & 0,060 & 1,261 & 0,074 \\
\hline 8 & 1,176 & 0,134 & 0,950 & 0,042 & & & & & & & & \\
\hline
\end{tabular}

D.S. = una desviación estándar

\section{Materia ORgánica en la COLUMna de agua}

Las concentraciones de MO en la columna de agua antes y después del lavado in situ se presentan en la Tabla 2, donde la concentración de seston orgánico en Pichagua tuvo una mediana de $0,003 \mathrm{~g} \mathrm{~L}^{-1}\left(0,002\right.$ a $\left.0,006 \mathrm{~g} \mathrm{~L}^{-1}\right)$, en Huelmo 0,004 $\mathrm{g} \mathrm{L}^{-1}$ $\left(0,002\right.$ a $\left.0,005 \mathrm{~g} \mathrm{~L}^{-1}\right)$ y en Alao Sur fue de $0,005 \mathrm{~g} \mathrm{~L}^{-1}(0,003$ a $0,017 \mathrm{~g} \mathrm{~L}^{-1}$ ). El test de Wilcoxon nos indica diferencias significativas en la concentración de seston entre todas las áreas de estudio $\left(P_{\text {Pichagua-Huelmo }}=0,022, P_{\text {Pichagua-Alao }}=1,95 \times 10^{-07}\right.$, $\left.P_{\text {Huelmo-Alao }}=0,0001\right)$, mientras que las diferencias dentro de las áreas, antes y después del lavado in situ de la concentración de seston en la columna de agua solo se manifiestan en el área de Pichagua y a los $5 \mathrm{~m}(P=0,039)$, siendo menor la concentración después del lavado (mediana de $0,004 \mathrm{~g} \mathrm{~L}^{-1}$ antes y $0,002 \mathrm{~g} \mathrm{~L}^{-1}$ después del lavado in situ). No se encontraron diferencias en la concentración de seston entre las profundidades por cada localidad $\left(P_{\text {pichagua 5-15 }}=0,64, P_{\text {huelmo 5-15 }}=0,16, P_{\text {alao 5-15 }}=\right.$ $0,46)$.

\section{Materia ORGánica EN SEDIMENTOS}

El porcentaje de materia orgánica de sedimentos asociados a los centros de cultivo estudiados antes y después del lavado in situ se muestran en la Tabla 3, donde la mediana en Pichagua fue de $0,99 \%$ (0,874 a 1,27\%), en Huelmo fue de 1,47\% (1,10 a 2,89\%) y Alao Sur fue de $1,29 \%$ (1,07 a 2,40\%) (Tabla 3 ). El análisis de Wilcoxon mostró diferencias significativas en 
cuanto al porcentaje de materia orgánica de los sedimentos entre Pichagua y Huelmo $\left(P=3,34 \times 10^{-6}\right)$, Pichagua y Alao Sur $\left(P=1,86 \times 10^{-5}\right)$, pero no entre Huelmo y Pichagua $(P=$ $0,328)$. No se encontraron diferencias en cuanto a los tratamientos dentro del área (Pichagua, $P=0,843$; Huelmo, $P=0,937$; Alao Sur, $P=0,156$ ) antes y después del lavado in situ.

\section{Granulometría}

Los porcentajes de grava, arena y materia orgánica, antes y después del lavado in situ, de los sedimentos asociados a los centros de cultivo, se presentan en la Tabla 4, en donde arena es la fracción dominante en los sedimentos de las 3 áreas o centros de cultivo, (93\% Pichagua, 97\% Huelmo, 94\% Alao Sur), seguido del fango (6\% Pichagua, 2\% Huelmo, $4 \%$ Alao Sur) y grava $(0,1 \%$ Pichagua, $0,4 \%$ Huelmo y $1.4 \%$ Alao Sur $)$ (Tabla 4). Al realizar un test de Wilcoxon sobre los porcentajes (aplicando transformación de arcoseno) de grava, arena y fango de los sedimentos asociados a los centros de cultivo, se apreció que entre las áreas existen diferencias entre arena de Pichagua y Huelmo $(P=0,0001)$ y entre Pichagua y Alao Sur $(P=$ $0,0001)$, pero no entre Huelmo y Alao Sur $(P=0,140)$. Para el fango existen diferencias solo entre Pichagua y Huelmo $(P=$ $0,001)$, pero no entre Pichagua y Alao $\operatorname{Sur}(P=0,140)$ ni entre Huelmo y Alao Sur $(P=0,140)$. En tanto para la grava se detectaron diferencias significativas entre Pichagua y Huelmo $(P=0,0001)$, y Pichagua y Alao Sur $(P=0,0001)$, pero no entre Huelmo y Alao $\operatorname{Sur}(P=0,140)$. Al analizar dentro de las áreas, se detectó que en Pichagua existen diferencias entre el antes y el después del lavado in situ en el porcentaje de fango $\left(P_{\text {fango }}=0,015\right.$, mayor después del lavado in situ $)$ y arena $\left(P_{\text {arena }}=0,015\right.$, mayor antes del lavado in situ $)$. No se encontraron diferencias granulométricas entre los otros lugares ni matrices analizadas.

\section{SALINIDAd, Temperatura y OXÍGENo}

La salinidad, temperatura y oxígeno, en promedio para las 3 áreas se muestran en la Figura 2, donde se observa que la salinidad presenta un patrón similar en relación a la profundidad entre las localidades estudiadas, con rangos que van de 33,5 a 33,6 en Pichagua, 31,2 a 32,8 en Huelmo y Alao Sur. Algo similar ocurrió con la temperatura donde los rangos fueron de 10,5 a $10,7^{\circ} \mathrm{C}$ en Pichagua, 10,6 a $11,2^{\circ} \mathrm{C}$ en Huelmo y Alao Sur, pero la concentración de oxígeno en la columna de agua presentó patrones diferentes en los primeros $10 \mathrm{~m}$, siendo similares los rangos de variación ( 6 a $7 \mathrm{mg} \mathrm{L}^{-1} \mathrm{deO}_{2}$, en Pichagua y de 6 a $9 \mathrm{mg} \mathrm{L}^{-1}$ de $\mathrm{O}_{2}$ en Huelmo y Alao Sur).

\section{Discusión}

Hasta el 2012 la medida de control de incrustantes más ampliamente usada ha sido el uso de pinturas anti incrustantes (Yebra et al. 2004, de Nys \& Guenther 2009, Dürr \& Watson 2010, Vera et al. 2015). Estas pinturas lixivian compuestos biocidas tales como metales pesados y biocidas orgánicos sobre las superficies, produciendo una fina capa tóxica que evita la fijación de incrustantes. Sin embargo, muchos de los químicos y metales pesados utilizados en estas pinturas son reconocidos como peligrosos para el medio ambiente, con efectos perjudiciales sobre la supervivencia y el crecimiento de moluscos (Paul \& Davies 1986 fide Fent 2006) y peces (Lee et al. 1985, Short \& Thrower 1986, Bruno \& Ellis 1988). Esto ha motivado a desarrollar un gran esfuerzo para prevenir o mitigar la contaminación biológica en acuicultura a través de métodos alternativos a las pinturas como el lavado in situ de redes.

Sin embargo, esta modalidad de impregnar podría ser cambiada en su totalidad a lavados in situ, puesto que algunos

Tabla 4. Porcentajes de grava, arena y fango, antes y después del lavado in situ, de los sedimentos asociados a los centros de cultivo / Percentages of gravel, sand and mud, before and after the in situ washing

\begin{tabular}{|c|c|c|c|c|c|c|c|c|c|c|c|c|c|c|c|c|c|c|}
\hline \multirow{4}{*}{ Estación } & \multicolumn{18}{|c|}{ Porcentaje de grava, arena y fango en sedimentos (Draga) } \\
\hline & \multicolumn{6}{|c|}{ Pichagua } & \multicolumn{6}{|c|}{ Huelmo } & \multicolumn{6}{|c|}{ Alao Sur } \\
\hline & \multicolumn{3}{|c|}{ antes } & \multicolumn{3}{|c|}{ después } & \multicolumn{3}{|c|}{ antes } & \multicolumn{3}{|c|}{ después } & \multicolumn{3}{|c|}{ antes } & \multicolumn{3}{|c|}{ después } \\
\hline & grava & arena & fango & grava & arena & fango & grava & arena & fango & grava & arena & fango & grava & arena & fango & grava & arena & fango \\
\hline 1 & 0,2 & 97,0 & 2,8 & 0,9 & 88,9 & 10,2 & 0,5 & 97,9 & 1,6 & 0,2 & 95,1 & 4,6 & 13,1 & 83,4 & 3,6 & 6,7 & 89,9 & 3,4 \\
\hline 2 & 0,0 & 95,7 & 4,2 & 0,0 & 94,7 & 5,3 & 0,3 & 99,4 & 0,3 & 0,2 & 99,5 & 0,3 & 0,0 & 97,8 & 2,2 & 0,0 & 96,6 & 3,3 \\
\hline 3 & 0,0 & 94,6 & 5,4 & 0,1 & 92,6 & 7,4 & 0,1 & 99,2 & 0,7 & 0,3 & 98,9 & 0,8 & 0,1 & 83,8 & 16,1 & 0,1 & 97,7 & 2,2 \\
\hline 4 & 0,0 & 96,4 & 3,6 & 0,0 & 96,5 & 3,5 & 0,1 & 99,2 & 0,7 & 0,1 & 100,1 & 0,0 & 0,0 & 97,9 & 2,1 & 0,1 & 99,5 & 0,5 \\
\hline 5 & 0,0 & 96,3 & 3,6 & 0,0 & 94,5 & 5,5 & 0,2 & 99,4 & 0,4 & 0,3 & 97,9 & 1,8 & 0,0 & 98,8 & 1,2 & 0,0 & 82,9 & 17,0 \\
\hline 6 & 0,1 & 95,0 & 4,9 & 0,1 & 91,2 & 8,7 & 0,2 & 98,1 & 1,7 & 0,4 & 97,9 & 1,7 & 0,0 & 96,7 & 3,3 & 0,1 & 98,4 & 1,6 \\
\hline 7 & 0,1 & 95,0 & 4,9 & 0,1 & 94,7 & 5,2 & 2,6 & 97,3 & 0,0 & 0,6 & 84,0 & 15,4 & 0,1 & 99,3 & 0,6 & 0,1 & 100,3 & 0,0 \\
\hline 8 & 0,1 & 87,3 & 12,6 & 0,1 & 85,5 & 14,4 & 0,1 & 94,8 & 5,0 & & & & & & & & & \\
\hline
\end{tabular}




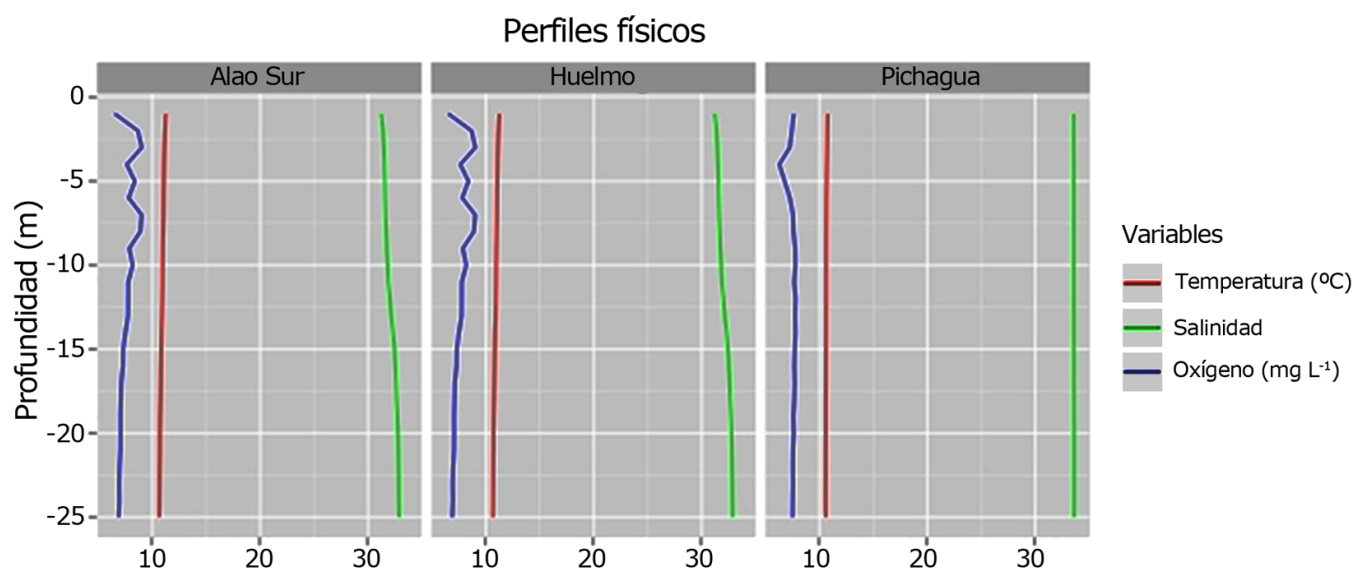

Figura 2. Salinidad, temperatura y oxígeno, en promedio para las 3 localidades estudiadas / Salinity, temperature and oxygen on average for the three locations studied

talleres de redes que lavan e impregnan, están insertando en la empresa salmonera a nivel experimental, nuevas fibras como el Euroline y redes de aleación de Cobre (Vera et al. 2015) en la confección de las redes loberas y peceras que no requieren de pinturas anti-incrustantes y son capaces de mantenerse en el agua relativamente limpias por más de 9 meses. Por otra parte, los lavados in situ de redes tienden a intensificarse en primavera y verano, debido al aumento de organismos incrustantes en las redes (Rubio et al. 2014). A esto se agrega la posibilidad de que algunos ciclos productivos que hayan utilizado redes impregnadas estén llegando a cosecha en esa misma época, realicen lavado in situ para evitar un nuevo proceso de impregnación, con los costos asociados a esto.

Este trabajo constituye el primer esfuerzo de evaluar el efecto del desprendimiento de incrustantes debido al lavado in situ de redes en empresas de salmónidos, en los ecosistemas del sur de Chile. Si se toma en cuenta la profundidad que existe en los centros de cultivo estudiados en esta ocasión (25-40 m) y la variabilidad producida por corrientes y mareas (Rodrigo 2006, Silva \& Astorga 2010), existe la posibilidad de que el material desprendido producto del tratamiento, que efectivamente es capaz de depositarse en el bentos, lo esté haciendo fuera del perímetro del centro de cultivo, producto de su tiempo de residencia en la columna de agua por lo que las trampas de sedimento en esta ocasión, solo capturan las partículas que sedimentan más rápido o las que a su vez son trasladadas desde otras localidades y que finalmente se depositan en las áreas en estudio (Lalande et al. 2016). En este proceso también influyen variables como la densidad de la partícula, forma de la partícula, velocidad de corrientes, densidad del agua, además de factores biológicos como el pastoreo (Vargas \& González 2004) y alimentación por parte del plancton de las partículas suspendidas en la columna de agua y la acción propia del anillo microbiano (Tsaia et al. 2013). Algunos de estos factores y su variación espacio-temporal son propios de cada lugar (González et al. 2004, Montecino \& Pizarro 2006) y pueden verse afectados por la estacionalidad (Iriarte et al. 2007, Daneri et al. 2012, Gutiérrez et al. 2016).

Debe hacerse notar que la cantidad de materia orgánica presente en los sedimentos asociados a los 3 centros de cultivo solo alcanzó un máximo de 2,9\%, lo que dista del $9 \%$ establecido por la normativa chilena vigente para considerar un sedimento como impactado por la acuicultura (RES.EXE. Subpesca $N^{\circ} 3612$, Art. 31) ${ }^{1}$, lo que hace pensar en la posibilidad de dispersión de las partículas en la columna de agua y su posterior traslado y sedimentación en zonas aledañas a los centros de cultivo, evitando así, alcanzar valores peligrosos de materia orgánica en los sedimentos bajo los centros de cultivo. Ya que en esta ocasión las trampas de sedimento solo fueron colocadas cerca de los centros de cultivo y no en gradiente alejándose de ellos, no fue posible determinar si existe depositación de material proveniente de la limpieza in situ en los alrededores de los centros.

Si se considera lo anterior, la granulometría de las 3 zonas de estudio nos muestra patrones similares, donde se aprecian diferencias entre los centros o áreas estudiadas, pero no dentro de ellas, predominando siempre en los 3 centros, el tipo de

${ }^{1}$ Resolución SUBPESCA N³612. 2009. Que fija las metodologías para elaborar la caracterización preliminar del sitio (CPS) y la información ambiental (INFA). Título IV De las variables, número 26. <http://www.subpesca.cl/normativa/605/w3-article-10517.html> 
sedimento arena, probablemente debido a la velocidad de la corriente (Cupul-Magaña et al. 2006). Si se toman en cuenta los resultados de la granulometría de Pichagua, se aprecia que dentro del lugar de muestreo, el porcentaje de arena es mayor antes del lavado in situ y luego de este disminuye, también varía el fango aumentando significativamente después del lavado indicando un efecto en los sedimentos que puede atribuirse al lavado in situ de redes y la sedimentación de material fino. Las otras localidades no presentaron este tipo de variación, por lo que nuevos ensayos son necesarios para validar la situación.

Los resultados obtenidos en este trabajo nos indican que las 3 zonas estudiadas, Pichagua, Huelmo y Alao Sur son en general distintas entre sí en cuanto a las tasas de sedimentación, seston y materia orgánica en sedimentos, pero dentro de cada localidad existen diferencias que parecen no estar relacionadas con el lavado in situ de redes, sino más bien con variabilidad propia de la zona como lo expresado por Weston (1986) quien concluye que la liberación de desechos provenientes de los bioincrustantes de las redes en salmonicultura, no son una fuente orgánica significativa sobre los sedimentos cercanos a las jaulas de salmones. Alao sur por ejemplo presenta una tasa de sedimentación mayor antes del lavado y no después. Lo contrario ocurre en Pichagua, donde la tasa de sedimentación y el seston fueron mayores después del lavado in situ. Otras matrices no mostraron diferencias.

Si bien el diseño experimental de este trabajo lleva a establecer diferencias debido al lavado in situ de redes, se debe considerar que las balsas-jaulas de salmónidos se encontraban activas y por lo tanto con régimen de alimentación (antes o después del lavado) y que los peces que se encuentran en el cultivo continúan produciendo fecas, por lo que existe la posibilidad que las diferencias encontradas se deban a estos factores y no al desprendimiento de incrustantes desde las redes debido al lavado. Otras estimaciones del total de sólidos suspendidos provenientes de la salmonicultura están entre 5 y $50 \mathrm{~g} \mathrm{~m}^{-2} \mathrm{~d}^{-1}$ (Merican \& Phillips 1985), mientras que en este caso van de 0,2 a $5 \mathrm{~g} \mathrm{~m}^{-2} \mathrm{~d}^{-1}$. Gowen \& Bradbury (1987) estimaron tasas de sedimentación de $27,4 \mathrm{~g} \mathrm{~m}^{-2} \mathrm{~d}^{-1}$ bajo salmoneras irlandesas y un promedio de $8,2 \mathrm{~g} \mathrm{~m}^{-2} \mathrm{~d}^{-1}$ en el perímetro de las balsas jaulas. Gowen et al. (1988) midieron tasas de sedimentación promedio de $82,2 \mathrm{~g} \mathrm{~m}^{-2} \mathrm{~d}^{-1}$ en el perímetro de las balsas-jaulas en Washington, mientras que Hardgrave (1994) revisó datos de tasas de sedimentación de otras áreas obteniendo un rango $1-181 \mathrm{~g}^{\mathrm{TOC}} \mathrm{m}^{-2} \mathrm{~d}^{-1}$ bajo el perímetro de balsas-jaulas operativas.

Brooks \& Mahnken (2003) no encontraron información sobre tasas de sedimentación de carbón asociado a balsas jaulas más allá del perímetro de las balsas jaulas. Sin embargo, Ye et al. (1991) asumió que estaciones de muestreo localizadas a
$150 \mathrm{~m}$ del perímetro representan condiciones de referencia, mientras que Croos (1990) y Holmer \& Kristensen (1992) asumen que la distancia puede ser mayor a $30 \mathrm{~m}$.

Si bien se encontraron diferencias en las matrices estudiadas entre los 3 sitios, solo se encontraron diferencias en las tasas de sedimentación dentro de Pichagua (menor antes que después del lavado) y Alao Sur, siendo esta última localidad una anomalía debido a que las tasas de sedimentación fueron mayores antes del lavado in situ. Pese a los resultados de este trabajo, aún es incierto que el lavado in situ tenga un efecto acumulativo a largo plazo sobre los sedimentos marinos, bajo o aledaños a los centros de cultivo, por lo que es necesario promover estudios y análisis de mayor duración temporal (mensuales, estacionales y/o anuales) de las tasas de sedimentación como los desarrollados por González et al. (2006), Lalande et al. (2016) y los de producción primaria por Weston et al. (2013), para obtener series de tiempo que puedan dar cuenta del real efecto de esta acción de limpieza propia de la acuicultura en los ecosistemas marinos del sur de Chile.

\section{Agradecimientos}

Se agradece al proyecto Subpesca 2012 'Evaluación ambiental de las actividades de lavado in situ en la acuicultura' (Res Exenta $\left.N^{\circ} 1575\right)$. También se agradece la participación de Daniela Gutiérrez, Adán Navarro y Joel Villalobos en los muestreos y en la recopilación de información para este trabajo.

\section{LITERATURA CITADA}

Beveridge M. 1987. Cage aquaculture, 352 pp. Fishing News Books, London.

Brooks K \& C Mahnken. 2003. Interactions of Atlantic salmon in the Pacific northwest environment II. Organic wastes. Fisheries Research 62: 255-293.

Bruno DW \& AE Ellis. 1988. Histopathological effects in Atlantic salmon, Salmo salar attributed to the use of tributyltin antifoulant. Aquaculture 72: 15-20.

Carl C, J Guenther \& LM Sunde. 2011. Larval release and attachment modes of the hydroid Ectopleura larynx on aquaculture nets in Norway. Aquaculture Research 42: 10561060.

Cronin ER, A C Cheshire, SM Clarke \& AJ Melville. 1999. An investigation into the composition, biomass and oxygen budget of the fouling community on tuna aquaculture farm. Biofouling 13: 279-299.

Cupul-Magaña LA, C Mösso-Aranda, JP Sierra, E Martí, JL Ferman-Almada, M Rodilla, J González del Río \& A Sánchez-Arcilla. 2006. Caracterización y patrones de distribución de los sedimentos superficiales en Bahía Cullera, España. Ciencias Marinas 32(4): 617-629. 
Daneri G, P Montero, L Lizárraga, R Torres, JL Iriarte, B Jacob, H González \& F Tapia. 2012. Primary productivity and heterotrophic activity in an enclosed marine area of central Patagonia (Puyuhuapi channel; $44^{\circ} \mathrm{S}, 73^{\circ} \mathrm{W}$ ). Biogeoscience Discussions 9: 5929-5968.

de Nys R \& J Guenther. 2009. The impact and control of biofouling in marine fin fish aquaculture. In: Hellio C \& D Yebra (eds). Advances in marine antifouling coatings and technologies, pp. 177-221. Woodhead Publishing, Cambridge.

Dürr S \& DI Watson. 2010. Biofouling and antifouling in aquaculture. In: Dürr S \& JC Thomason (eds). Biofouling, pp. 267-287. Wiley-Blackwell, Oxford.

Fent K. 2006. Worldwide occurrence of organotins from antifouling paints and effects in the aquatic environment. In: Konstantinou I(ed). Antifouling paint biocides. The Handbook of Environmental Chemistry 5: 71-100. Springer-Verlag, Berlin

Fitridge I, T Dempster, J Guenther \& R de Nys. 2012. The impact and control of biofouling in marine aquaculture: a review. Biofouling 28(7): 649-669.

González H, D Hebbeln, JL Iriarte \& M Marchant. 2004. Downward fluxes of faecal material and microplankton at $2300 \mathrm{~m}$ depth in the oceanic area off Coquimbo $\left(30^{\circ} \mathrm{S}\right)$, Chile, during 1993-1995. Deep-Sea Research II 51: 2457-2474.

González HE, G Daneri, E Menschel, M Marchant, S Pantoja, D Hebbeln, C Barria, R Vera \& J Iriarte. 2006. Downward fluxes of particulate organic matter in coastal and oceanic areas off Chile: The role of the OMZ and functional groups of the plankton. Gayana 70(Supl. 1): 26-28.

Gowen RJ \& NB Bradbury. 1987. The ecological impact of salmonid farming in coastal waters: a review. Oceanography and Marine Biology Annual Review 25: 563-575.

Gowen, RJ, J Brown, N Bradbury \& DS McLusky. 1988. Investigation into benthic enrichment, hypernutrification and eutrophication associated with mariculture in Scottish coastal waters (1984-1988). Department of Biological Sciences, University of Stirling, Scotland.

Greene JK \& RE Grizzle. 2007. Successional development of fouling communities on open ocean aquaculture fish cages in the western Gulf of Maine, USA. Aquaculture 262: 289301.

Guenther J, C Carl \& LM Sunde. 2009. The effects of colour and copper on the settlement of the hydroid Ectopleura larynx on aquaculture nets in Norway. Aquaculture 292: 252255.

Guenther J, E Misimi \& LM Sunde. 2010. The development of biofouling, particularly the hydroid Ectopleura larynx, on commercial salmon cage nets in Mid-Norway. Aquaculture 300: 120-127.

Gutiérrez D, M Akester \& L Naranjo. 2016. Productivity and sustainable management of the Humboldt Current large marine ecosystem under climate change. Environmental Development 17: 126-144.
Hodson SL \& C Burke. 1994. Microfouling of salmon cage netting: a preliminary investigation. Biofouling 8: 93-105.

Hodson SL, TE Lewis \& CM Burke. 1997. Biofouling of fish cage netting: efficacy and problems of in situ cleaning. Aquaculture 152: 77-90.

Holmer M \& E Kristensen. 1992. Impact of fish cage farming on metabolism and sulfate reduction on the underlying sediments. Marine Ecology Progress Series 80: 191-201.

Hopkins GA, BM Forrest, RF Piola \& JP Gardner. 2011. Factors affecting survivorship of defouled communities and the effect of fragmentation on establishment success. Journal of Experimental Marine Biology and Ecology 396: 233-243.

Iriarte JL, H González, KK Liu, C Rivas \& C Valenzuela. 2007. Spatial and temporal variability of chlorophyll and primary productivity in surface waters of southern Chile (41.5$43^{\circ} \mathrm{S}$ ). Estuarine, Coastal and Shelf Science 74: 471-480.

Lalande C, EM Nöthig, E Bauerfeind, K Hardge, A Beszczynska-Möller \& K Fahl. 2016. Lateral supply and downward export of particulate matter from upper waters to the seafloor in the deep eastern Fram Strait. Deep Sea Research I 114: 78-89.

Lee HB, LC Lim \& L Cheong. 1985. Observations on the use of antifouling paint in net cage fish farming in Singapore. Singapore Journal of Primary Industries 13: 1-12.

Menton DJ \& JH Allen. 1991. Spherical (Kiel) and square steelcages: first year of comparative evaluations at St. Andrews, NB. Bulletin of the Aquaculture Association of Canada 91: 111-113.

Merican ZO \& MJ Phillips. 1985. Solid waste production from rainbow trout (Salmo gairdneri Richardson) cage culture. Aquatic Fisheries Management 1: 55-69.

Montecino V \& G Pizarro. 2006. Productividad primaria, biomasa y tamaño del fitoplancton en canales y fiordos australes: patrones primavera-verano. In: Silva N \& S Palma (eds). Avances en el conocimiento oceanográfico de las aguas interiores chilenas, Puerto Montt a cabo de Hornos, pp. 9397. Comité Oceanográfico Nacional, Pontificia Universidad Católica de Valparaíso, Valparaíso.

Needham T. 1988. Sea water cage culture of salmonids. In: Laird LM \& T Needham (eds). Salmon and trout farming, pp. 117-154. Ellis Horwood, Chichester.

Olafsen T. 2006. Cost analysis of different antifouling strategies, 23 pp. SINTEF Fisheries and Aquaculture, SFH80A066041.

Rodrigo C. 2006. Caracterización y clasificación de la bahía de Puerto Montt mediante batimetría de multihaz y datos de backscatter. Investigaciones Marinas 34(1): 83-94.

Rubio D, C López-Galindo, J Casanueva \& E Nebot. 2014. Monitoring and assessment of an industrial antifouling treatment. Seasonal effects and influence of water velocity in an open once-through seawater cooling system. Applied Thermal Engineering 67(1-2): 378-387. 
Short JW \& FP Thrower. 1986. Accumulation of butyltin in mussel of Chinook salmon reared in sea pens treated with tri-n-butyltin. Marine Pollution Bulletin 17: 542-545.

Silva N \& MI Astorga. 2010. Textura, materia orgánica y composición química elemental $(\mathrm{C}$ y $\mathrm{N})$ de sedimentos marinos superficiales de la zona Puerto Montt a Boca del Guafo (Norpatagonia chilena). Latin American Journal of Aquatic Research 38(1): 1-14.

Tsaia AY, G-C Gonga \& YW Huanga. 2013. Variations of microbial loop carbon flux in western subtropical Pacific coastal water between warm and cold season, Journal of Experimental Marine Biology and Ecology 449: 111-117.

Vargas CA \& HE González. 2004. Plankton community structure and carbon cycling in a coastal upwelling system. II. Microheterotrophic pathway. Aquatic Microbial Ecology 34: $165-180$.

Vera R, C Duarte, E Pinilla, V Murillo, M Oyarzún \& G Aroca. 2015. Determinación y evaluación de los componentes presentes en las pinturas anti-incrustantes utilizadas en la acuicultura y sus posibles efectos en sedimentos marinos en el sur de Chile. Latin American Journal of Aquatic Research 43(2): 351-366.
Weston DP. 1986. The environmental effects of floating mariculture in Puget Sound, 148 pp. Washington Department of Fisheries and the Washington Department of Ecology, Olympia.

Weston KJ, D Timothy, C Damien, A Clarke, M Meredith, MI Wallace, MA Brandon, SJ Ussher \& KR Hendry. 2013. Primary production export flux in Marguerite Bay (Antarctic Peninsula): Linking upper water-column production to sediment trap flux. Deep-Sea Research I: 75: 52-66.

Ye L-X, DA Ritz, GE Fenton \& ME Lewis. 1991. Tracing the influence on sediments of organic waste from a salmonid farm using stable isotope analysis. Journal of Experimental Marine and Biological Ecology 145: 161-174.

Yebra DM, S Kiil \& K Dam-Johansen. 2004. Antifouling technology - past, present and future steps towards efficient and environmentally friendly antifouling coatings. Progress in Organic Coatings 50:75-104. 